\title{
La Investigación Psicosocial Actual Referida a la Salud Mental de las Personas Transgénero: Una Mirada Desde Chile
}

\section{Current Psychosocial Research on Transgender Individuals' Mental Health: A Chilean Perspective}

\author{
Jaime Barrientos \\ Universidad Alberto Hurtado \\ José L. Saiz \\ Universidad de La Frontera \\ Fabiola Gómez \\ Pontificia Universidad Católica de Chile \\ Mónica Guzmán-González y Ricardo Espinoza-Tapia \\ Universidad Católica del Norte \\ Manuel Cárdenas \\ Universidad de Valparaíso \\ Joaquin Bahamondes \\ University of Auckland
}

\begin{abstract}
Las personas transgénero han logrado progresivamente visibilidad y derechos en muchas sociedades occidentales. La investigación psicosocial sobre dicha población es reciente y se focaliza en el estudio del prejuicio sexual y su impacto sobre la salud mental de las personas transgénero. En este artículo se busca clarificar algunas definiciones conceptuales usadas en la investigación psicosocial, sintetizar la evidencia obtenida en Chile y observar cómo esta dialoga con la literatura internacional. Si bien el foco es la salud mental, se examinan también los efectos del prejuicio y el rechazo social como precursores de una salud mental deteriorada. Este artículo pretende contribuir al debate e investigación en temas conceptuales y metodológicos aún pendientes referidos a la población transgénero en América Latina.
\end{abstract}

Palabras clave: transgénero, salud mental, psicosocial

\begin{abstract}
Transgender individuals have progressively acquired visibility and rights in many western societies. Psychosocial research on this population is recent and has focused on sexual prejudice and its impact on the mental health of transgender people. This paper intends to clarify some conceptual definitions used in psychosocial research, summarize the evidence obtained in Chile, and observe how it relates to international literature. Although the article focuses on mental health, the effects of prejudice and social rejection as precursors of a deteriorated mental health are also examined. This paper aims to contribute to debate and research on pending conceptual and methodological issues related to transgender populations in Latin America.
\end{abstract}

Keywords: transgender, mental health, psychosocial

En América Latina aún persiste un conjunto de inequidades que afectan las relaciones de género y que son sostenidas por las ideologías del patriarcado y el heterosexismo (Barrientos, 2015). Dichas ideologías sustentan, por un lado, la dominación social que los hombres ejercen sobre las mujeres (patriarcado) y, por otro, al estatus privilegiado de las relaciones románticas heterosexuales por sobre aquellas que no lo son (heterosexismo). Estas desigualdades tendrían un impacto adverso en diversos ámbitos de la vida de quienes no se ajustan a las normas y expectativas de género impuestas por dichas ideologías (Barrientos, 2015).

Jaime Barrientos, Escuela de Psicología, Universidad Alberto Hurtado, Santiago, Chile; José L. Saiz, Departamento de Psicología, Universidad de La Frontera, Temuco, Chile; Fabiola Gómez, Escuela de Psicología, Pontificia Universidad Católica de Chile, Santiago, Chile; Mónica Guzmán-González y Ricardo Espinoza-Tapia, Escuela de Psicología, Universidad Católica del Norte, Antofagasta, Chile; Manuel Cárdenas, Escuela de Psicología, Universidad de Valparaíso, Chile; Joaquin Bahamondes, School of Psychology, Faculty of Science, University of Auckland, Nueva Zelanda.

Este artículo fue financiado por la Comisión Nacional de Investigación Científica y Tecnológica de Chile, a través del Proyecto FONDECYT N 1170046.

La correspondencia relativa a este artículo debe ser dirigida a Jaime Barrientos, Escuela de Psicología, Universidad Alberto Hurtado, Avda. Libertador Bernardo O'Higgins 1869, Santiago, Chile. E-mail: jbarrientos@uahurtado.cl 
En particular, tanto el patriarcado como el heterosexismo favorecen un conjunto de condiciones adversas para quienes se definen como lesbianas, gays, bisexuales, transgénero e intersexuales (en adelante, LGBTI; Barrientos, 2015; Herek, 2000) y promueven un modelo binario del género (masculino o femenino; McCann \& Sharek, 2016). En la cultura occidental, todas aquellas personas que no encajan en este modelo binario han sido y son frecuentemente consideradas por la sociedad como desviadas (Coleman et al., 2012). La caracterización de las sexualidades no heterosexuales como desviadas es propia de las ciencias de la salud y, particularmente, de la psiquiatría y la sexología (Barrientos, 2015). Tal es el caso de las personas transgénero (en adelante, personas trans), quienes, al igual que otras minorías sexuales, no encajan en el modelo binario del género.

En los últimos años, ha aumentado el debate público sobre las personas trans (Halberstam, 2018; Stryker, 2017), lo que ha estimulado el surgimiento de un campo de-discusión, investigación y trabajo para las ciencias sociales y para la psicología en particular. Este campo de estudios ha tenido un desarrollo más fecundo en otros países de América Latina, como Argentina (Arrubia \& Brocca, 2017; Fundación Huésped, 2017; Rada Schultze, 2016; Rigueiral \& Seidmann, 2016, Noviembre), Brasil (Costa et al., 2015; de Souza, Malvasi, Signorelli \& Pereira, 2015) o Perú (Pollock, Silva-Santisteban, Sevelius \& Salazar, 2016; Silva-Santisteban et al., 2012), mientras que en otros países, como Chile, el debate sobre las personas trans es más reciente y la investigación psicosocial sobre el tema es aún escasa, comparada con la de otros países de América Latina (Barrientos, 2015). No obstante, a nivel nacional, se destacan algunos trabajos recientes, como los de Barreda e Isnardi (2006), Berredo de Toledo Lobato (2011), Casanova Bahamondes y Espinoza-Tapia (2018), Muñoz, Zamorano y Alvarado (2009) o el de Valdés y Riquelme (2016).

A pesar de la mayor visibilidad e interés por estudios sobre las personas trans, en Latinoamérica no existen revisiones en español actualizadas de la literatura científica internacional sobre aspectos relativos a la salud mental de las personas trans. La mayor cantidad de investigación existente sobre el tema de la salud de las personas trans y su relación con el prejuicio ha sido hecha en Estados Unidos y está disponible únicamente en inglés. Por otro lado, en el debate e investigación sobre el tema, aún existe bastante imprecisión conceptual sobre las personas trans, tanto en el resto del mundo como en América Latina, aunque recientemente comienza a escribirse más sobre el tema (Aguirre-Sánchez-Beato, 2018). En este sentido, este artículo pretende contribuir a la superación de algunas de esas debilidades, enfatizando los efectos del prejuicio sexual en la salud mental de las personas trans.

Además de avanzar en la clarificación de definiciones conceptuales usadas en la investigación con población trans, este artículo aporta hallazgos obtenidos hasta ahora en Chile (especialmente, de aquellos estudios hechos mediante encuestas), vinculándolos con algunos ejemplos de la literatura internacional (incluyendo en esta última a la latinoamericana). Si bien el foco de este artículo es la salud mental de la población trans desde una mirada psicosocial, se examinará también el prejuicio y el rechazo social como precursores de una salud mental deteriorada. Finalmente, se quisiera contribuir con algunas preguntas que enriquezcan el debate sobre temas conceptuales y metodológicos aún pendientes. No obstante, se debe precisar que, en aras a la extensión de este artículo, se priorizarán algunas tradiciones teóricas más que otras. De esta forma, por ejemplo, el modelo de estrés de minorías (en adelante, MEM) de Ilan Meyer (2003) será, sin duda, una referencia obligada en desmedro de otros.

\section{Definiciones Conceptuales Relativas a las Personas Trans}

Se comenzará por definir y clarificar brevemente algunos términos relevantes que se usan frecuentemente en la discusión e investigación sobre las personas trans. Contar con definiciones conceptuales nítidas y diferenciadas indudablemente contribuirá al avance en el conocimiento científico de esta población. Además, tal como lo ha enfatizado Aguirre-Sanchéz-Beato (2018), es complejo definir a las personas trans y, por ende, estudiar la discriminación hacia dichas personas. Considerando que el foco de este artículo no es la historia de los conceptos, lamentablemente, se hará una referencia parcial a dichos aspectos históricos. Para mayor detalle sobre este punto, se sugiere revisar otros trabajos en los que la historia conceptual es ampliamente desarrollada (por ejemplo, ver Stryker, 2017).

Si bien diferentes conceptos (por ejemplo, transexual o transgénero) han sido usados para enfatizar diferentes definiciones asociadas a las personas trans (Aguirre-Sanchéz-Beato, 2018), la palabra transgénero conecta, al menos, dos nociones importantes: sexo e identidad de género. Pese a ser términos diferentes, durante mucho tiempo estos han sido usados de modo intercambiable, generando una gran confusión conceptual e ingentes dificultades en la investigación y discusión en el área (Stryker, 2017). De hecho, el 
concepto de sexo se ha utilizado inicialmente y durante mucho tiempo como única categorización de la población trans y solo en la década del 50 surge el concepto de género, en parte gracias al trabajo de John Money (1955) y Robert Stoller (1964), lo cual permite contemplar una dimensión no biológica de la sexualidad y la identidad.

El sexo, generalmente, es asignado por el médico al nacer (o durante la ecografía), de acuerdo a la apariencia de los genitales externos. No obstante, cuando los genitales externos son ambiguos, se considera que otros indicadores (por ejemplo, los genitales internos o el sexo cromosómico y hormonal) permiten asignar el sexo (American Psychological Association [APA], 2015).

A diferencia del sexo, de fundamento claramente biológico, el género es un término socialmente construido que refiere a la distinción social o cultural asociada con un determinado sexo. Estrechamente vinculado al género, está el concepto de identidad de género, el cual alude a la sensación subjetiva de carácter profundo e intenso que tiene una persona de ser un hombre, una mujer o una persona de un género alternativo (denominada de género no conforme o neutral). Esta sensación puede corresponderse o no con el sexo asignado al nacer o a las características sexuales primarias o secundarias de una persona (APA, 2015).

Considerando las definiciones previas, en este artículo se usará la noción de transgénero para definir a aquellas personas cuya identidad de género o la expresión de esta difiere de lo esperado culturalmente, una vez que se ha hecho la asignación de sexo al nacer como hombre o mujer (Davidson, 2007; Valentine, 2007). El término transgénero parece imponerse en la literatura internacional, pese a las diferencias en la vivencia individual de la experiencia transgénero en el plano de los contextos locales (Valentine, 2007). Por su parte, el término cisgénero será empleada para aludir a aquellas personas cuya identidad de género y el sexo asignado al nacer son concordantes con el comportamiento que a esta persona le fue socialmente asignado (APA, 2015).

Es importante agregar que la noción de identidad de género es diferente a la de orientación sexual. Cuando comenzó a usarse el término transgénero, se pensaba que una persona trans (identidad de género) era una persona homosexual (orientación sexual; Stryker, 2017). No obstante, hoy estos términos están claramente diferenciados, al menos en la investigación psicosocial, lo que no evita que aún, a veces, se siga usando como equivalente la noción de orientación sexual homosexual con la de transgénero. El concepto de orientación sexual se refiere a un componente específico de la identidad, que incluye la atracción sexual y emocional de una persona hacia otra persona y el comportamiento y/o la relación social que pueden resultar de esta atracción (APA, 2015). Por tanto, una persona puede sentirse atraída por hombres, mujeres, por ninguno de los dos o por personas que son andróginas (persona cuya expresión de género es una mezcla de la expresión de género masculina y femenina). Así, los individuos pueden identificarse como lesbianas (mujeres atraídas por mujeres), gay (hombres atraídos por hombres), heterosexuales (personas atraídas por el sexo opuesto), bisexuales (personas atraídas por ambos sexos), asexuales (personas no atraídas por ningún sexo) o pansexuales (personas atraídas por otras, sin importar la orientación sexual de estas últimas).

Una complejidad conceptual adicional estriba en que las personas trans suelen definir de diferentes maneras su identidad de género. Por ejemplo, algunas personas trans deciden hacer solo una transición social, cambiando su nombre o su expresión de género. En cambio, otras deciden hacer tanto una transición social, la que puede incluir o no intervenciones corporales, así como una transición médica y, consecuentemente, se someten a terapia hormonal y/o recurren a cirugía de conformidad de género u otra intervención quirúrgica (por ejemplo, implante de mamas) para hacer concordante su expresión de género con su identidad. Esto se ha relacionado claramente con las posibilidades que las personas trans poseen en cuanto al acceso a tratamientos y a la atención de especialistas en distintos países (Organización Panamericana de la Salud [OPS], 2016).

Un aspecto muy relevante, considerado también en la literatura, es la diferenciación entre personas trans femeninas, esto es, aquellas cuyo sexo asignado al nacer era varón, pero que actualmente se identifican como mujer y las personas trans masculinas, esto es, aquellas cuyo sexo asignado al nacer era femenino, pero que se identifican actualmente como hombres (APA, 2015). Además, hay personas trans que eligen tener una identidad y/o expresión de género fuera del tradicional modelo sexual binario y de género (Davidson, 2007), es decir, optan por un movimiento permanente entre lo masculino/femenino, autodenominándose personas de género fluido (gender fluid). Este artículo no abordará este grupo.

Igualmente, una palabra ampliamente usada en la literatura científica y particularmente en la literatura médica es la de transexual. Este término ha sido y sigue siendo usado, desde las ciencias biomédicas y psiquiátricas, para describir a las personas trans que han cambiado o están cambiando sus cuerpos a través 
de intervenciones médicas (por ejemplo, hormonas o cirugía), con la finalidad de "alinear" sus cuerpos con una identidad de género que es diferente de su sexo asignado al nacer. El término transexual, por tanto, se utiliza como una categorización diagnóstica en el lenguaje biomédico, por lo que el uso de verbos como alinear enfatiza la idea de que existe un desajuste que debe resolverse mediante una intervención biomédica. Por tanto, el uso de este término, y generalmente el diagnóstico asociado, se aplica a las personas que presentan, desde dicha óptica biomédica, una incongruencia o falta de conformidad entre el sexo que les fue asignado al momento de nacer de acuerdo a sus caracteres sexuales y la identidad autopercibida. Al respecto, es importante añadir que, desde la identidad autopercibida, no todas las personas que se identifican como transexuales se consideran trans. Por ejemplo, algunas personas transexuales se identifican simplemente como mujeres u hombres sin recurrir a una identidad trans. Además, cabe añadir que la palabra transexual ha sido utilizada largamente en el diagnóstico médico según la Clasificación Internacional de Enfermedades (CIE-10) de la Organización Mundial de la Salud (APA, 2015), considerando la transexualidad como un trastorno de la identidad de género, posición que ha sido largamente criticada en la literatura. Esta noción ha sufrido sucesivas modificaciones en estos manuales diagnósticos: desde transexualismo, pasando por trastorno de identidad de género, hasta llegar a la reciente disforia de género.

Por su parte, en América Latina es aún de uso común la palabra travesti. Este término tiene su origen en una categoría diagnóstica presente en manuales de psiquiatría y hace referencia a aquellas personas que usan ropa que convencionalmente es propia del sexo opuesto, pero que no emprenden ningún tipo de proceso de modificación anatómica permanente. En algunos países como Brasil y Chile, el término es ampliamente usado como equivalente a la actual noción de transgénero. Sin embargo, su uso suele ser más frecuente para referirse a hombres que transitan hacia lo femenino que para las mujeres que transitan hacia lo masculino y, además, suele ser una palabra muy estigmatizada (Stryker, 2017). Por ello, en el mundo angloparlante, según autoras como Stryker (2017), se usa el término cross-dresser como sustituto no moralizante de la palabra travesti. Recientemente, en países como Argentina o Chile, algunas activistas reivindican el uso de la palabra travesti para visibilizar otros marcadores de diferencia, además de la identidad, como los de clase o etnia (Rodríguez, 2018, Agosto).

Otro concepto importante a definir, ya que es comúnmente utilizado en el debate e investigación sobre este tema, es el de disforia de género, término que ha sido incluido en la última edición del Manual Diagnóstico y Estadístico de los Trastornos Mentales (DSM-V) de la Asociación Americana de Psiquiatría (Stryker, 2017). Este concepto, desde una óptica biomédica, por un lado, hace referencia a la disconformidad afectiva/cognitiva de un individuo con el género asignado y, por otro, alude al malestar que podría acompañar la inconsistencia entre el género experimentado o expresado y aquel asignado a un individuo cuando se usa como categoría diagnóstica.

También, es necesario agregar que en la investigación social reciente sobre las personas trans la noción de transgénero se ha operacionalizado de varias formas. Según Reisner et al. (2016) esas formas pueden ser sintetizadas en dos aproximaciones: una, basada en la identidad de género ligada a la autopercepción (en las que se usa la noción de transgénero) y, otra, basada en un diagnóstico psiquiátrico o clínico (en las que se usa la noción de transexualidad o disforia de género). Sin embargo, según las autoras mencionadas, actualmente existiría una comprensión basada en la identidad de género, noción que es consistente con la creciente idea de la despatologización de la diversidad sexual y de género (Dellacasa, 2017), a la vez que el reconocimiento de las propias definiciones que las personas trans usan para referirse a sí mismas (Aguirre-Sánchez-Beato, 2018).

\section{Recomendaciones para el Trabajo con Personas Trans}

Para estandarizar la recopilación de datos sobre personas trans y producir información relevante para la investigación y la intervención, actualmente se ha recomendado el uso de un enfoque que supone considerar dos dimensiones para la toma de decisión, atendiendo al contexto geográfico específico, el idioma y la configuración regional particular (ver Tabla 1; Reisner et al., 2016). Este enfoque, que considera conjuntamente el sexo asignado al nacer y la identidad de género autopercibida, permitiría avanzar en el monitoreo y evaluación de los esfuerzos para lograr una mayor equidad en salud (Reisner et al., 2016). 
Tabla 1

Método de Dos Pasos para Estandarizar la Recopilación de Datos en Estudios con Población Trans

\begin{tabular}{lcc}
\hline \multirow{2}{*}{ Identidad de género actual } & \multicolumn{2}{c}{ Sexo asignado al nacer } \\
\cline { 2 - 3 } & Hombre & Mujer \\
\hline Masculina & Cisgénero & Trans \\
Femenina & Trans & Cisgénero \\
Trans masculino/trans de mujer hacia hombre & No aplica & Trans \\
Trans femenina/trans de hombre hacia mujer & Trans & No aplica \\
Genderqueer/género no conforme & Trans & Trans \\
\hline
\end{tabular}

\section{Investigación sobre la Situación de Personas Trans}

\section{Prevalencia de las Personas Trans}

Aunque no existen datos precisos sobre el tamaño de la población trans en el mundo, las estimaciones más recientes sugieren una prevalencia de 0,3 a $0,5 \%$ de personas que se identifican como trans (Reisner et al., 2014). En Chile, los datos del Ministerio de Desarrollo Social, recopilados en 2015 mediante la Encuesta de Caracterización Socioeconómica Nacional, aportan información chilena sobre la prevalencia de las personas trans (Instituto Nacional de Derechos Humanos [INDH], 2017). Según dichos datos, se estima que un $2,3 \%$ de la población chilena mayor de 18 años es transmasculina, mientras que un 3,1\% de la población mayor de 18 años es transfemenina. Sin embargo, considerando las dificultades de acceso a esta población y que las estimaciones difieren en función de la definición de transgénero que se emplee, es probable que el tamaño de esta población esté subreportada en los diferentes trabajos (Meier \& Labuski, 2013). Frente a esta incerteza, la literatura empírica sobre prevalencia de personas trans recomienda adherirse a definiciones específicas de la condición de transgénero, a fin de asegurar la comparabilidad de los resultados (Collin, Reisner, Tangpricha \& Goodman, 2016).

\section{Prejuicio Sexual hacia las Personas Trans}

El prejuicio sexual orientado hacia las personas trans se ha denominado transfobia. No obstante, para los efectos del presente artículo se usará la expresión de prejuicio sexual en vez de transfobia, ya que ese término es el más usado en la investigación psicosocial sobre diversidad sexual y existe más consenso en su uso (Fraïssé \& Barrientos, 2016).

Herek (2000) define originalmente el prejuicio sexual como una actitud negativa hacia otras personas basada en la orientación sexual de estas últimas, prejuicio que casi siempre está dirigido hacia gais, lesbianas, bisexuales o personas trans. Más recientemente, este término se ha usado más ampliamente para aludir a toda actitud negativa hacia personas no heterosexuales (Barrientos, 2015). Además, en el caso de las personas trans, este prejuicio sexual se combina y profundiza con una actitud de rechazo hacia los cuerpos y el aspecto de las personas trans (por ejemplo, la vestimenta).

El prejuicio sexual es un complejo y dinámico proceso que se manifiesta tanto a nivel estructural, como interpersonal e individual. El nivel estructural se refiere a las normas sociales y las políticas institucionales que restringen el acceso a recursos o derechos. Por ejemplo, la patologización y medicalización de las personas trans representa una de las principales y más importantes formas de prejuicio sexual estructural. Este tipo de prejuicio moldea y refuerza las percepciones sociales de las personas trans como desviadas (Dellacasa, 2017). El nivel interpersonal se refiere a las formas directas de prejuicio sexual, como la violencia física o sexual debido a la identidad de género o su expresión, puesto que, desde la perspectiva prejuiciosa, estas últimas transgredirían las expectativas de género. Por último, el nivel individual incluye la percepción que las propias personas trans tienen del prejuicio del que son objeto, así como la autopercepción negativa y los sentimientos negativos que pueden derivar de esta (Reisner, Hughto et al., 2015; Reisner, Pardo et al., 2015). 
Actualmente, en muchos países, aún la situación respecto de los derechos de las minorías sexuales es muy negativa, siendo mucho peor aún para las personas trans (Chiam, Duffy \& González Gil, 2017).

Estudios recientes señalan que los niveles de prejuicio sexual hacia la población trans son más elevados que aquellos existentes hacia otras minorías sexuales (Barrientos, 2015), reportándose, además, numerosos crímenes de odio hacia personas trans en el mundo (Barrientos, Cárdenas \& Saiz, 2016; International Lesbian, Gay, Bisexual, Trans and Intersex Association [ILGA], 2017).

En Chile se ha avanzado bastante en cuanto a derechos relativos a las minorías sexuales. Actualmente, estos están más instalados en la agenda pública, especialmente luego de la aprobación de una ley antidiscriminación en 2012 y del Acuerdo de Unión Civil en 2015, un contrato que permite la vida en pareja entre personas de cualquier orientación sexual. En particular, la Ley de Identidad de Género, luego de cinco años de discusión parlamentaria, fue promulgada el año 2018. Esta ley faculta a que personas trans sobre los 14 años puedan cambiar su nombre legal y sexo registral, sin necesidad de realizar intervenciones quirúrgicas, como es el caso de la cirugía de confirmación de género. Finalmente, se puede mencionar la firma del Acuerdo por la Igualdad el año 2016 entre el Estado y la Comisión Interamericana de Derechos Humanos, abarcando temáticas de salud y educación en políticas públicas que benefician a la diversidad sexual (INDH, 2017).

Reflejando la precaria situación a nivel global de las personas trans, la Oficina del Alto Comisionado de las Naciones Unidas para los Derechos Humanos (s.f.) ha denunciado la discriminación generalizada hacia ellas, la marginación social y económica sistemática, el estigma, la patologización, la discriminación, la violencia y otras violaciones de derechos humanos, incluidas las de atención médica. Además, en la misma línea y refiriéndose a la disciplina psicológica, en Estados Unidos la APA (2009) encontró que menos del 30\% de los psicólogos estaba familiarizado con la situación de las personas trans. Asimismo, se ha encontrado que los profesionales de la salud tienen limitada experiencia con esta población, pudiendo con ello causar daño con las intervenciones que realizan (Mikalson, Pardo \& Green, 2013).

Pese a los avances en materias legislativas en Chile, sigue siendo importante conocer el estado actual de las actitudes hacia las personas trans. Un reciente estudio efectuado por la ILGA (2017) en 75 países del mundo, aporta información relevante al respecto. Dicho estudio encuestó a 116.000 personas en todo el mundo y a 4.234 en Chile. A nivel internacional se reporta que 50\% de los encuestados está de acuerdo en que a los adultos que se visten, actúan o se identifican con un sexo, aunque nacieran con otro, se les debe otorgar el pleno reconocimiento legal de la identidad que declaran, mientras que el $25 \%$ está en desacuerdo y el $25 \%$ no está de acuerdo ni en desacuerdo. Además, parece haber una clara asociación entre aceptar la protección de igualdad de derechos y reconocer legalmente el género: quienes están de acuerdo con la protección tienden también a estar de acuerdo con el reconocimiento.

Los datos recolectados en Chile por este mismo estudio indican que un $38 \%$ de los encuestados conoce al menos a una persona que se viste, actúa o se identifica como una persona trans, mientras que un $50 \%$ no conoce a nadie y un $11 \%$ no sabe. Además, dicho estudio consultó sobre el grado de acuerdo/desacuerdo con diversas afirmaciones referidas a las personas trans, tal como se puede observar en la Tabla 2.

Asimismo, otro reciente estudio internacional realizado por Ipsos (2018), en el que se realizaron entrevistas en una muestra de 27 países ( $n=19747$ adultos), aporta datos interesantes para la situación de Chile. En el país se observa que un $48 \%$ de los entrevistados ha visto a personas trans, pero no les conoce personalmente, mientras que un $13 \%$ tiene un amigo o familiar trans. Además, un $61 \%$ de los entrevistados cree que ser trans es algo natural, mientras que solo un $13 \%$ de los chilenos cree que la condición trans se debe a algún tipo de enfermedad mental. Por último, cabe destacar que, según este estudio, Chile es el segundo país, luego de Argentina, donde las personas están mayoritariamente de acuerdo (84\%) con que las personas trans deberían estar protegidas contra la discriminación por parte del gobierno. También, Chile es el primer país, de aquellos estudiados, donde se observa un fuerte apoyo a las operaciones quirúrgicas que mejoren la situación de las personas trans (82\%). Por último, seis de cada 10 personas entrevistadas en todo el mundo (59\%) creen que su país se está volviendo más tolerante con las personas trans. Esta percepción es más fuerte en Argentina (78\%), Canadá (78\%) y Chile (76\%). 
Tabla 2

Grado de Acuerdo de los Chilenos con las Afirmaciones Referidas a Personas Trans

\begin{tabular}{lcc}
\hline \multicolumn{1}{c}{ Afirmación } & $\begin{array}{c}\text { Ni de } \\
\text { acuerdo } \\
\text { ni en } \\
\text { Algo o muy en } \\
\text { desacuerdo } \\
\text { desacuerdo }\end{array}$ & $\begin{array}{c}\text { Algo o muy de } \\
\text { acuerdo }\end{array}$ \\
\hline $\begin{array}{l}\text { La igualdad de derechos y protecciones deberían ser } \\
\text { aplicados a todos, incluyendo a la gente que se viste, actúa o } \\
\text { identifica como de un sexo, aunque hayan nacido con otro. }\end{array}$ & $11 \%$ & $14 \%$ \\
$\begin{array}{l}\text { Los adultos que se visten, actúan o se identifican con un solo } \\
\text { sexo a pesar de que nacieron con otro: ise les debe otorgar } \\
\text { pleno reconocimiento legal de la identidad que declaran? }\end{array}$ & $11 \%$ & $21 \%$ \\
$\begin{array}{l}\text { Es posible respetar mi cultura y aceptar a las personas que } \\
\text { se visten, actúan o identifican con un solo sexo, aunque } \\
\text { nacieron con otro. }\end{array}$ & $10 \%$ & $75 \%$ \\
\hline
\end{tabular}

Fuente: ILGA (2017).

Nota. Para este artículo se han sumado las opciones muy en desacuerdo y algo en desacuerdo y las opciones algo de acuerdo y muy de acuerdo.

En Chile hay poca investigación acumulada sobre prejuicio sexual hacia las personas trans que incorpore una óptica psicosocial. No obstante, en el año 2017 la agrupación Organizando Trans Diversidades (OTD) realizó en este país el primer estudio a gran escala sobre esta población. Mediante una encuesta online (Encuesta-T), en la que participaron 315 personas que se definieron como trans, indagó en diversos temas, entre ellos, el prejuicio sexual. Uno de los hallazgos relevantes se refiere a las experiencias de rechazo social: las personas encuestadas reportaron que al interior de la familia se viven experiencias intensas de estigma y prejuicio sexual, siendo la violencia el hecho más recurrente en dicho contexto, y el cuestionamiento de su identidad. Además, un 40\% de las personas encuestadas señaló haber sufrido violencia en su lugar de estudio y un 95\% declaró haber sentido que cuestionaban su identidad de género en centros de salud.

La revisión anterior parece indicar que los cambios legislativos han redundado en actitudes más positivas hacia las personas trans en la población chilena general, al menos en un nivel declarativo. Esta situación, sin embargo, no es coherente con la experiencia de las propias personas trans, quienes informan altos niveles de estigma y prejuicio social (Barrientos, 2015).

\section{Salud Mental en Personas Trans}

Hasta hace algunos años atrás, la investigación psicosocial sobre salud mental en personas trans había sufrido un importante retraso, debido a la clasificación de lo trans como un trastorno mental (Coll-Planas, 2010). La literatura ha reportado dos posiciones antagónicas al respecto: una, pro-derechos de las personas trans, que procura eliminar la lógica diagnóstica psiquiátrica del Manual Diagnóstico y Estadístico de los Trastornos Mentales (DSM-V; American Psychiatric Association, 2014) y otros similares, como el manual de Clasificación Internacional de Enfermedades (CIE-10; World Health Organization, 1992) y, otra posición, de naturaleza conservadora, que procura mantener la clasificación de las identidades trans como trastornos mentales (Coll-Planas, 2010). La persistencia aún de este debate sobre el carácter patológico o no de las identidades trans ha dificultado la discusión e investigación sobre la salud mental de dicha población.

Sin embargo, recientemente la American Psychiatric Association, en su última versión del DSM, ha desestimado la inclusión del llamado trastorno de identidad de género, nombre con el que, hasta ahora, se tipificaba la condición transexual. Según las nuevas normas del DSM-V, las personas trans solo reciben un diagnóstico denominado disforia de género cuando la persona experimenta malestar por la incongruencia entre la propia experiencia de género y el asignado al nacer.

El argumento de la despatologización de lo trans pone en el tapete una de las principales paradojas de la medicalización del diagnóstico de esta condición, tal como ha sido indicado claramente por Dellacasa (2017), quien plantea que el retiro de la categorización de disforia de género del DSM quita la noción de patología adscrita a la condición de transgénero. Sin embargo, la autora añade que, si bien por un lado, la eliminación de la disforia de género de dichos manuales diagnósticos tendría enormes beneficios para las personas trans, 
por otro, podría inducir a la salud pública a desligarse de la obligación de cubrir los costos de los tratamientos hormonales y de las cirugías de reasignación, lo que podría ocasionar muchos problemas para las mismas personas trans. Esta tensión a la que se refiere Dellacasa (2017) existe, debido a la persistencia de la ubicación de la condición trans en el plano de la patología.

La exposición a prejuicio sexual ha sido asociada a un deterioro de la salud en personas trans, incluyendo depresión, ansiedad, suicidio, abuso de sustancias y virus de inmunodeficiencia humana (VIH), entre otras (OPS, 2016). También, se ha documentado que el prejuicio sexual es responsable de indicadores negativos en la salud de las personas trans, ya sea actuando directamente como fuente de estrés o indirectamente, restringiendo el acceso tanto a diversos recursos protectores de la salud como a conocimiento pertinente (Link \& Phelan, 2006). Además, en muchos contextos, las personas trans carecen de acceso a cuidados de salud físicos y mentales que reconozcan y respalden su identidad/expresión de género, lo que se configura como una gran barrera de acceso a la salud (Fredriksen-Goldsen et al., 2014).

Dada la situación descrita, la población trans posee un mayor riesgo que gais, lesbianas y población cisgénero de experimentar disparidades de salud (Su et al., 2016). Dichas dificultades para el acceso a la salud hacen posible que las personas trans tengan mayor prevalencia que las personas cisgénero de sufrir depresión, angustia, suicidio y otros desórdenes mentales, debido al mayor nivel de estrés social provocado por el prejuicio sexual asociado la condición trans (Bockting et al., 2016).

En relación con lo anterior, la OPS (2016) ha elaborado un completo listado de los principales problemas relacionados con la salud de la población trans en América Latina: (a) altos niveles de exposición a violencia verbal, emocional y física, incluyendo los crímenes de odio; (b) alta frecuencia de problemas relacionados con la salud mental (depresión y ansiedad); (c) alta tasa de prevalencia de VIH y otras infecciones de transmisión sexual; (d) alto consumo de alcohol y drogas; (e) efectos negativos de hormonas autoadministradas, inyecciones para rellenar diversas zonas del cuerpo y otras formas de modificación corporal, incluyendo complicaciones por malas intervenciones de reasignación de sexo, y (f) problemas de salud reproductiva.

La mayor parte de los estudios psicosociales sobre la salud en población trans usa indicadores negativos de salud física y mental (Barrientos, 2015). Sin embargo, el prejuicio sexual también puede generar consecuencias negativas sobre indicadores positivos de salud mental, como el bienestar psicológico y social, siendo solo muy recientes los estudios que abordan estos indicadores positivos en minorías sexuales y casi inexistentes en población trans (Barrientos, 2015). Si bien actualmente la salud mental es una de las áreas más investigadas en la población trans (Marshall, Claes, Bouman, Witcomb \& Arcelus, 2016), aún queda mucho por conocer. En tal sentido, se requiere avanzar en la descripción, caracterización y comprensión de las inequidades en salud existentes entre las personas trans y las personas cisgénero, así como en la investigación de los factores que favorecen y limitan la salud mental de la población trans.

Aunque recientemente ha aumentado la investigación en general sobre las personas trans en América Latina y Chile, lamentablemente, aún hay poca evidencia empírica sobre los mecanismos mediante los cuales el prejuicio sexual afecta la salud mental de dicha población. La mayor parte de la evidencia que revela los efectos negativos del prejuicio sexual en la salud física y mental de las personas trans ha sido generada en poblaciones de Estados Unidos y Europa (Reisner et al., 2016), siendo más escasos los estudios que documenten dicha asociación en América Latina (Barrientos Delgado \& Cárdenas Castro, 2014; Casanova Bahamondes \& Espinoza-Tapia (2018; Costa et al., 2015; Fundación Huésped, 2017; OPS, 2016; Rada Schultze, 2016; Reisner et al., 2016).

Un modelo teórico que permite articular coherentemente la diversa evidencia sobre prejuicio sexual y disparidades en salud en minorías sexuales es el MEM (Meyer, 2003). El MEM señala que las minorías sexuales estarían expuestas a múltiples fuentes adicionales de estrés en razón de su pertenencia grupal. Estas fuentes, que incluyen, entre otras, eventos de discriminación, expectativas de rechazo, ocultación de la identidad sexual y rechazo internalizado (Meyer, 2003), impactarían negativamente la salud de los miembros de las minorías sexuales. Este modelo, que incorpora también factores estructurales, interpersonales e individuales, ha generado abundante evidencia empírica (Dunn, Gonzalez, Costa, Nardi \& Iantaffi, 2014).

Aunque en Chile la investigación sobre salud mental de las personas trans es escasa, se dispone de algunos datos. La Encuesta-T (OTD, 2017), ya mencionada, indagó también sobre salud de las personas trans. Entre los principales hallazgos, se encontró que un 56\% declaró haber intentado suicidarse alguna vez en su vida. Además, un alto porcentaje de las personas encuestadas manifestó deseos de realizar cambio de nombre $(76,5 \%)$ y sexo legal $(76,2 \%)$. Dicha encuesta fue realizada el 2017, un año antes de la aprobación de la ley de identidad de género en Chile. Asimismo, este estudio también constató el deseo de las personas trans por 
acceder a cirugías de feminización o masculinización, así como por tener acceso a tratamientos hormonales. Por ejemplo, un $75 \%$ de las personas encuestadas que querían hacer el tránsito de masculino a femenino declaró que desearía someterse a cirugías de feminización y un $80 \%$ de las personas que deseaban hacer el tránsito de femenino a masculino manifestó que anhelaría someterse a cirugías de masculinización.

La información previamente descrita por la Encuesta-T es concordante con los escasos estudios psicosociales previos hechos en población trans en el país (Barrientos \& Bozon, 2014; Barrientos Delgado \& Cárdenas Castro, 2014; Barrientos, Cárdenas, Gómez \& Guzmán, 2016). Otros estudios psicosociales hechos en Chile han encontrado información diferente a la ya descrita, pero complementaria. Por ejemplo, un estudio realizado por el Centro de Estudios de la Sexualidad y el Movimiento Unificado de Minorías Sexuales (2009), en población transfemenina que ejerce el comercio sexual, reveló que estas personas poseen una gran vulnerabilidad social, especialmente respecto al VIH. Otro estudio comparó a hombres gais y a población trans femenina, encontrando que los gais reportaban mayores niveles de percepción de felicidad que las personas transfemeninas (Barrientos Delgado \& Cárdenas Castro, 2014).

\section{Limitaciones y Perspectivas en la Investigación sobre las Personas Trans}

Un reporte reciente de la OPS (2016) indica que existen enormes vacíos en el conocimiento sobre las personas trans en América Latina. Tales vacíos se refieren a la ausencia de caracterizaciones sociodemográficas y sociales de la población trans, de datos sobre epidemiología y problemas de salud, de estudios sobre prevalencia de comportamientos de riesgo y de predictores de riesgo y resiliencia. Dado que la investigación en el campo es aún limitada, se requiere un cuerpo nuevo de evidencia que pueda servir de base para la asignación de recursos y disminuir las disparidades de salud existentes en esta población.

Parece indudable que los fenómenos psicosociales están condicionados por los contextos culturales en los que ocurren. Al estar la investigación sobre población trans altamente concentrada en Estados Unidos y, más recientemente, en Europa (Reisner et al., 2016), el conocimiento allí generado no necesariamente es generalizable a otros entornos culturales. Se hace, por tanto, necesario abordar el estudio de esta población desde perspectivas locales, identificando similitudes y diferencias respecto del conocimiento previamente establecido.

Otro aspecto relevante a considerar en futuras investigaciones es que los diferentes perfiles que puede asumir la vivencia de cada persona trans (ya sea se trate de travestis, trans, transexuales, personas sometidas o no a intervención hormonal y/o quirúrgica) están escasamente documentados (Valentine, 2007). Por tanto, se requiere avanzar en la identificación precisa de dichos perfiles.

Cabe mencionar que la mayoría de los estudios hechos hasta la fecha referidos a personas trans evalúan el uso y abuso de sustancias, la salud sexual y sus problemas de salud mental (Reisner et al., 2016). No obstante, dichos estudios han puesto escaso énfasis en otros temas igualmente relevantes, como los problemas de acceso a una adecuada asistencia sanitaria, así como los patrones de uso de dicha asistencia, las complicaciones derivadas del tratamiento hormonal y de los procedimientos quirúrgicos a los que se someten muchas personas trans y las tasas de enfermedades crónicas relacionadas con la edad y el envejecimiento (que se cree se ven afectadas por la exposición hormonal), entre otros (Reisner et al., 2016).

Adicionalmente, la mayor parte de la investigación que se ha realizado con población trans generalmente se ha basado en el uso de muestras por conveniencia, siendo la mayoría de los estudios publicados en Estados Unidos de tipo transversal o retrospectivo (Reisner et al., 2016). Se hace, por tanto, necesario avanzar en el empleo de muestras representativas a fin de garantizar la generalización de los resultados. De utilidad podrían ser técnicas de muestreo que permiten la obtención de muestras representativas en poblaciones cuyo tamaño es impreciso o desconocido, como es el caso de las minorías sexuales (Cárdenas Castro \& Yáñez Yáñez, 2012). Otra necesidad es el empleo de diseños longitudinales, a fin de establecer la evolución de los fenómenos bajo estudio.

Además, la indagación debería considerar que las condiciones de salud de este grupo están entrañablemente ligadas a su particular estatus social bajo dentro de la sociedad; omitir este vínculo resultaría problemático y solo contribuiría a la profundización del estigma social. Asimismo, la falta de encuestas estandarizadas y específicamente orientadas a estudiar a las personas trans, así como la dificultad de acceso a esta población, limita mucho los esfuerzos existentes respecto de los aspectos relativos a la salud de dicha población. Estos problemas solo son subsanables en la medida en que exista un genuino diálogo con las agrupaciones y personas trans, las cuales deben ser incorporadas a los equipos y tratadas en calidad de expertas. Este último aspecto es coherente con las recomendaciones hechas por Staples, Bird, Masters y 
George (2017) para el estudio sobre las personas trans. Estas recomendaciones incluyen, entre otras: (a) proporcionar a los participantes una justificación clara del estudio; (b) considerar durante la creación de encuestas el impacto emocional que pueden tener ciertos temas sensibles; (c) no limitar las opciones de respuesta, ofreciendo la posibilidad de respuestas abiertas; (d) evitar el uso de un lenguaje binario referido a la identidad; (e) evaluar separadamente la conducta sexual de la orientación sexual y (f) involucrar, siempre que sea posible, a los miembros de la comunidad trans en todas las etapas de la investigación.

La literatura plantea que una mejora en la salud y en el acceso a la atención médica de las personas trans requiere una amplia gama de partes interesadas y la movilización de diversas asociaciones multisectoriales (Reisner et al., 2016). También señala que una característica fundamental de la investigación con personas trans es que esta sea centrada en el sujeto (Reisner et al., 2016). Consecuentemente, la indagación con dicha población requiere involucrar, desde el principio, a los miembros locales de las propias comunidades trans, lo que asegurará que el diseño del estudio y los métodos de recopilación de datos sean factibles y aceptables para todos los participantes. De esa forma, los estudios de salud en población trans probablemente se beneficiarían de la aplicación de protocolos de investigación basados en la comunidad para trabajar con personas trans en el diseño, implementación y diseminación de investigación y no meramente en la población trans.

Lo anterior demuestra cómo en el contexto local existen aún bastantes vacíos en la investigación sobre la salud de las personas trans que deben ser llenados.

\section{Discusión y Conclusiones}

Este artículo busca incentivar la exploración psicosocial respecto de la situación de las personas trans en Chile y en América Latina. Usar una mirada psicosocial implica articular diferentes niveles de análisis (en este caso, niveles individual y social) y abordar el conflicto que surge en el encuentro entre el individuo y la sociedad. Igualmente, este artículo invita a interrogar a la psicología como disciplina y a los usos que se ha hecho de esta para legitimar el prejuicio sexual hacia las personas LGBTI y específicamente a las personas trans. Particularmente, busca interrogar a un cierto tipo de producción científica en psicología social sobre las personas trans, producida mediante encuestas, debido a su carácter acrítico, individual y atomizante. Esta crítica generalmente se ha hecho desde otros aportes recientes de corte crítico (Roselló-Peñaloza, 2018), aunque es importante precisar que este artículo no se ubica en dicha tradición crítica, sino más bien en una perspectiva psicosocial más clásica.

En ese sentido, la psicología y las ciencias psicológicas (entre las que están la psiquiatría y el psicoanálisis) han contribuido, conscientes o no, a la patologización de las identidades trans mediante el uso de diversas categorías diagnósticas (usadas aún en manuales como el DSM o el CIE) y que han sido usadas para justificar la discriminación hacia las personas trans. Esta mirada individualista promueve la definición de la condición trans como un problema que afecta a un grupo particular o a individuos concretos, sin considerar el origen y la función social del prejuicio sexual. Hoy en día, se discute la despatologización de tal condición y las organizaciones trans ejercen activismo en este sentido en el mundo entero.

Aún persisten tanto en la literatura como en los programas de salud abordajes diversos a nivel conceptual sobre la situación de las personas trans en el mundo y en Chile. Tal es el caso de que, si bien las dimensiones de sexo y género son importantes determinantes de la salud física y mental en una amplia variedad de situaciones, ambas categorías suelen solaparse al ser empleadas en investigación sobre el tema. Por ejemplo, los términos para referirse al sexo asignado al nacer (varón y mujer) y la identidad de género (hombres y mujeres) se usan indistintamente en la literatura científica, incluida la investigación referida a población trans. Esta práctica de solapamiento impide establecer si las diferencias de salud encontradas entre población trans y cisgénero se deben, efectivamente, al sexo, al género, a ambas categorías o a ninguna de las dos, lo que perjudica una comprensión cabal de las inequidades en salud que afectan a esta población.

Otro ejemplo a destacar en el ámbito de la intervención consiste en la comprensión sobre la experiencia trans mediante normativas en salud, como es el caso de las circulares $\mathrm{N}^{\circ} 34$ (Chile, Ministerio de Salud [MINSAL], 2011) y No21 (MINSAL, 2012) emanadas del Ministerio de Salud de Chile. En la primera de ellas, si bien se invita al personal de salud a respetar el uso del nombre social y apariencia física del paciente para efectos de hospitalización, se plantea que las personas trans tendrían un trastorno de identidad sexual, según clasificaciones pertenecientes al DSM-IV y CIE-10. La circular $\mathrm{N}^{\circ} 21$ (MINSAL, 2012) plantea un abordaje de las personas trans en el contexto sanitario desde un enfoque centrado en los derechos humanos de dicha comunidad. 
Adicionalmente, las diferencias encontradas en la forma de operacionalizar la condición trans y la escasa comprensión de las múltiples identidades y experiencias que comprende dicho concepto, dificultan aún más la investigación. Al mismo tiempo, el predominio de una mirada aún patologizante y centrada solo en indicadores negativos de salud mental ha promovido una acumulación de conocimiento sobre las personas trans que puede entorpecer la comprensión de las experiencias de estas desde una mirada psicosocial.

En otro sentido, la baja prevalencia de personas trans en el mundo y en Chile no debería hacer descuidar que estas personas están expuestas a diversas situaciones sociales, culturales y legales que les sitúa en una posición de vulnerabilidad frente a diversas manifestaciones de violencia, tal como el prejuicio sexual, que incluye crímenes de odio, y los efectos que este ocasiona en su salud mental.

Respecto de la contraparte empírica que debe tener la integración conceptual, Reisner et al. (2016) sugieren poner a prueba los modelos teóricos existentes que intentan explicar las disparidades respecto a deficiencias en salud entre diversos tipos de poblaciones o bien desarrollar nuevos modelos que permitan guiar la investigación relativa a indicadores positivos de salud y bienestar en la población trans. Tal como se indicó, se han aplicado varios modelos conceptuales, entre ellos el MEM. Este modelo y otros reconocen la existencia de múltiples niveles entrecruzados en la definición del riesgo y resiliencia que moldean la salud y bienestar de las personas trans, concordando en que es siempre necesario un abordaje multinivel contextualmente relevante (Reisner et al., 2016).

Por tanto, este artículo busca entregar elementos para la discusión de quienes hacen investigación psicológica y psicosocial a adoptar una perspectiva comprehensiva y más respetuosa de los derechos humanos de dichas personas. En particular, este trabajo deja abierta una invitación a los investigadores psicosociales a incorporar una comprensión sobre la diversidad sexual no patologizada y centrada en las particularidades de las identidades trans.

\section{Referencias}

Aguirre-Sánchez-Beato, S. (2018). Trans terminology and definitions in research on transphobia: A conceptual review. Quaderns de Psicologia, 20, 295-305. https://doi.org/10.5565/rev/qpsicologia.1453

American Psychiatric Association (2014). Manual diagnóstico y estadístico de los trastornos mentales: DSM-5 (5a ed.). Madrid, España: Médica Panamericana.

American Psychological Association (2009). Report of the APA Task Force on Gender Identity and Gender Variance. Washington, DC: Autor. Extraído de https://www.apa.org/pi/lgbt/resources/policy/gender-identity-report.pdf

American Psychological Association (2015). Guidelines for psychological practice with transgender and gender nonconforming people. American Psychologist, 70, 832-864. https://doi.org/10.1037/a0039906

Arrubia, E. \& Brocca, M. (2017). La construcción del estigma como límite a los derechos sociales de las personas trans desde una perspectiva internacional. Anuario de Derechos Humanos, 13, 87-96. Doi: 10.5354/0718-2279.2017.46891

Barreda, V. \& Isnardi, V. (2006). Travestis y prevención del VIH/SIDA: un escenario de categoría en crisis. En A. Piscitelli, C. F. Cáceres \& Seminario Regional "Salud, Sexualidad y Diversidad en América Latina" (Eds.), Sexualidad, estigma y derechos humanos: desafios para el acceso a la salud en América Latina (pp. 167-176). Lima, Perú: Universidad Peruana Cayetano Heredia, Facultad de Salud Pública y Administración.

Barrientos, J. (2015). Violencia homofóbica en América Latina y Chile. Santiago, Chile: El Buen Aire.

Barrientos, J. \& Bozon, M. (2014). Discrimination and victimization against gay men and lesbians in Chile: Two patterns or just one? Interdisciplinaria, 31, 323-339. https://doi.org/10.16888/interd.2014.31.2.8

Barrientos, J., Cárdenas, M., Gómez, F. \& Guzmán, M. (2016). Gay men and male-to-female transgender persons in Chile: An exploratory quantitative study on stigma, discrimination, victimization, happiness and social well-being. En T. Köllen (Ed.), Sexual orientation and transgender issues in organizations: Global perspectives on LGBT workforce diversity (pp. 253-270). Cham, Suiza: Springer.

Barrientos, J., Cárdenas, M. \& Saiz, J. L. (2016). Hate crimes in Chile. En E. Dunbar, A. Blanco \& D. Crèvecoeur-MacPhail (Eds.), The psychology of hate crimes as domestic terrorism: U.S. and global issues (Vol. 2, pp. 145-170). Santa Barbara, CA: Praeger.

Barrientos Delgado, J. \& Cárdenas Castro, M. (2014). Construction and validation of a subjective scale of stigma and discrimination (SISD) for the gay men and transgender women population in Chile. Sexuality Research and Social Policy, 11, 187-198. https://doi.org/10.1007/s13178-014-0150-0

Berredo de Toledo Lobato, L. (2011). Dificultades administrativas enfrentadas por las personas trans en la Región Metropolitana de Chile (Tesina de Grado, Universidad Academia de Humanismo Cristiano, Santiago, Chile). Extraído de http://bibliotecadigital.academia.cl/bitstream/handle/123456789/2188/TPERIO\%2096.pdf? sequence=1\&isAllowed=y

Bockting, W., Coleman, E., Deutsch, M. B., Guillamon, A., Meyer, I., Meyer, W. ... Ettner, R. (2016). Adult development and quality of life of transgender and gender nonconforming people. Current Opinion in Endocrinology, Diabetes and Obesity, 23, $188-197$. https://doi.org/10.1097/MED.0000000000000232

Casanova Bahamondes, P. \& Espinoza-Tapia, R. (2018). Significados en torno a la atención psicológica durante el proceso de transición de género: una aproximación desde la perspectiva de usuarios/as transgénero. Al Sur de Todo: Revista Multidisciplinaria de Género $y$ Cultura, 12, 105-116. Extraído de http://www.alsurdetodo.com/wp-content/uploads/2018/10/12.-Casanova-Bahamondes-yEsponiza-Tapia-2018-Significados-atención-durante-el-proceso-de-transición.pdf

Cárdenas Castro, M. \& Yáñez Yáñez, S. (2012). Nuevas formas de muestreo para minorías y poblaciones ocultas: muestras por encuestado conducido en una población de inmigrantes sudamericanos. Universitas Psychologica, 11, 571-578. https://doi.org/10.11144/Javeriana.upsy11-2.nfmm 
Centro de Estudios de la Sexualidad \& Movimiento Unificado de Minorías Sexuales (2009). Caracterización de la vulnerabilidad individual y grupal de personas trans de la Región Metropolitana, con énfasis en aquellas que ejercen el comercio sexual. Santiago, Chile: Autores. Extraído de http://www.scribd.com/doc/113015091/

Chiam, Z., Duffy, S. \& González Gil, M. (2017). Trans legal mapping report 2017: Recognition before the law. Genève, Suiza: International Lesbian, Gay, Bisexual, Trans and Intersex Association. Extraído de http://ilga.org/downloads/ILGA_Trans_Legal_Mapping_Report_2017_ENG.pdf

Chile, Ministerio de Salud (2011). Circular $N^{\circ} 34$. Instruye sobre la atención de personas trans y fortalecimiento de la estrategia de hospital amigo a personas de la diversidad sexual en establecimientos de la red asistencial. Santiago, Chile: Autor. Extraído de https://diprece.minsal.cl/wrdprss_minsal/wp-content/uploads/2015/01/CIRCULAR-34-Atenci\%C3\%B3n-de-personas-trans.pdf

Chile, Ministerio de Salud (2012). Circular $N^{\circ} 21$. Reitera instrucción sobre la atención de personas trans en la red asistencial. Santiago, Chile: Autor. Extraído de https://diprece.minsal.cl/wrdprss_minsal/wp-content/uploads/2015/01/CIRCULAR-21-ReiteraAtenci\%C3\%B3n-de-personas-trans.pdf

Coleman, E., Bockting, W., Botzer, M., Cohen-Kettenis, P., DeCuypere, G., Feldman, J. ... Zucker, K. (2012). Standards of care for the health of transsexual, transgender, and gender-nonconforming people, version 7. International Journal of Transgenderism, 13, 165232. https://doi.org/10.1080/15532739.2011.700873

Collin, L., Reisner, S. L., Tangpricha, V. \& Goodman, M. (2016). Prevalence of transgender depends on the "case" definition: A systematic review. The Journal of Sexual Medicine, 13, 613-626. https://doi.org/10.1016/j.jsxm.2016.02.001

Coll-Planas, G. (2010). Introducción. En M. Misse \& G. Coll-Planas (Eds.), El género desordenado: críticas en torno a la patologización de la transexualidad (pp. 9-22). Barcelona, España: Egales.

Costa, A. B., Fontanari, A. M. V., Jacinto, M. M., da Silva, D. C., Lorencetti, E. K., da Rosa Filho, H. T. ... Lobato, M. I. R. (2015). Population-based HIV prevalence and associated factors in male-to-female transsexuals from southern Brazil. Archives of Sexual Behavior, 44, 521-524. https://doi.org/10.1007/s10508-014-0386-z

Davidson, M. (2007). Seeking refuge under the umbrella: Inclusion, exclusion, and organizing within the category transgender. Sexuality Research and Social Policy, 4(4), 60-80. https://doi.org/10.1525/srsp.2007.4.4.60

Dellacasa, M. A. (2017). Una mirada arqueológica de los discursos sobre transexualidad: modalidades de producción de conocimiento y subjetividades. Psicoperspectivas, 16(3), 17-28. https://doi.org/10.5027/psicoperspectivas-vol16-issue3-fulltext-1053

de Souza, M. H. T., Malvasi, P., Signorelli, M. C. \& Pereira, P. P. G. (2015). Violência e sofrimento social no itinerário de travestis de Santa Maria, Rio Grande do Sul, Brasil [La violencia y el sufrimiento social en el itinerario de las personas transgénero de Santa María, Río Grande do Sul, Brasil]. Cadernos de Saúde Pública, 31, 767-776. https://doi.org/10.1590/0102-311X00077514

Dunn, T. L., Gonzalez, C. A., Costa, A. B., Nardi, H. C. \& Iantaffi, A. (2014). Does the minority stress model generalize to a non-US sample? An examination of minority stress and resilience on depressive symptomatology among sexual minority men in two urban areas of Brazil. Psychology of Sexual Orientation and Gender Diversity, 1, 117-131. https://doi.org/10.1037/sgd0000032

Fraïssé, C. \& Barrientos, J. (2016). Le concept d'homophobie: Une perspective psychosociale [El concepto de homofobia: una aproximación psicosocial]. Sexologies, 25, 133-140. https://doi.org/10.1016/j.sexol.2016.02.001

Fredriksen-Goldsen, K. I., Simoni, J. M., Kim, H. -J., Lehavot, K., Walters, K. L., Yang, J. ... Muraco, A. (2014). The health equity promotion model: Reconceptualization of lesbian, gay, bisexual, and transgender (LGBT) health disparities. American Journal of Orthopsychiatry, 84, 653-663. https://doi.org/10.1037/ort0000030.

Fundación Huésped (2017). Análisis de la accesibilidad y la calidad de atención de la salud para la población lesbiana, gay, trans y bisexual (LGBT) en cinco regiones sanitarias de la provincia de Buenos Aires: informe de resultados. Buenos Aires, Argentina: Autor. Extraído de https://www.huesped.org.ar/wp-content/uploads/2017/04/Informe-Salud-LGBT-PciaBsAs-FINAL.pdf

Halberstam, J. (2018). Trans*: A quick and quirky account of gender variability. Oakland, CA: University of California Press.

Herek, G. M. (2000). The psychology of sexual prejudice. Current Directions in Psychological Science, 9, 19-22. https://doi.org/10.1111/1467-8721.00051

Instituto Nacional de Derechos Humanos (2017). Realidad y desafío: niños, niñas y adolescentes trans e intersex en contextos de salud y educacionales. En Instituto Nacional de Derechos Humanos, Informe anual: situación de los derechos humanos en Chile (pp. 4251). Santiago, Chile: Autor.

International Lesbian, Gay, Bisexual, Trans and Intersex Association (2017). Minorities report 2017: Attitudes to sexual and gender minorities around the world. Genève, Suiza: Autor.

Ipsos (2018). Global attitudes toward transgender people. Paris, Francia: Autor. Extraído de https://www.ipsos.com/sites/default/files/ct/news/documents/2018-01/ipsos_report-transgender_global_data-2018.pdf

Link, B. G. \& Phelan, J. C. (2006). Stigma and its public health implications. The Lancet, 367, 528-529. https://doi.org/10.1016/S01406736(06)68184-1

Marshall, E., Claes, L., Bouman, W. P., Witcomb, G. L. \& Arcelus, J. (2016). Non-suicidal self-injury and suicidality in trans people: A systematic review of the literature. International Review of Psychiatry, 28, 58-69. https://doi.org/10.3109/09540261.2015.1073143

Meier, S. C. \& Labuski, C. M. (2013). The demographics of the transgender population. En A. K. Baumle (Ed.), International handbook on the demography of sexuality (pp. 289-327). New York, NY: Springer.

Meyer, I. H. (2003). Prejudice, social stress, and mental health in lesbian, gay, and bisexual populations: Conceptual issues and research evidence. Psychological Bulletin, 129, 674-697. https://doi.org/10.1037/0033-2909.129.5.674

Mikalson, P., Pardo, S. \& Green, J. (2013). First, do no harm: Reducing disparities for lesbians, gay, bisexual, transgender, queer and questioning populations in California. San Francisco, CA: Mental Health America of Northern California/California Mental Services Authority.

McCann, E. \& Sharek, D. (2016). Mental health needs of people who identify as transgender: A review of the literature. Archives of Psychiatric Nursing, 30, 280-285. https://doi.org/10.1016/j.apnu.2015.07.003

Money, J. (1955). Hermaphroditism, gender and precocity in hyperadrenocorticism: Psychological findings. Bulletin of The Johns Hopkins Hospital, 96, 253-264.

Muñoz, F., Zamorano, P. \& Alvarado, P. (2009). Caracterización del trabajo sexual en la Región Metropolitana (mujeres, hombres que tienen sexo con hombres y transgéneros). Santiago, Chile: Centro de Estudios de la Sexualidad/Moviemiento por la Diversidad Sexual/Fundación Margen.

Oficina del Alto Comisionado de las Naciones Unidas para los Derechos Humanos (s.f.). Personas lesbianas, gays, bisexuales, transgénero e intersex. Genève, Suiza: Autor. 
Organización Panamericana de la Salud (2016). Por la salud de las personas trans: elementos para el desarrollo de la atención integral de personas trans y sus comunidades en Latinoamérica y el Caribe. Buenos Aires, Argentina: Autor. Extraído de http://www.paho.org/arg/images/gallery/Blueprint\%20Trans\%20Espa $\tilde{A} \pm$ ol.pdf

Organizando Trans Diversidades (2017). Informe ejecutivo Encuesta T. Santiago, Chile: Autor. Extraído de encuesta-t.cl

Pollock, L., Silva-Santisteban, A., Sevelius, J. \& Salazar, X. (2016). 'You should build yourself up as a whole product': Transgender female identity in Lima, Peru. Global Public Health, 11, 981-993. https://doi.org/10.1080/17441692.2016.1167932

Rada Schultze, F. (2016). Situación laboral y condiciones de trabajo de las travestis en el Área Metropolitana de Buenos Aires. Red Sociales, 4, 67-90. Extraido de http://www.redsocialesunlu.net/wp-content/uploads/2017/03/RSOC018-007-Situaci\%C3\%B3nlaboral-y-condiciones-de-trabajo-de-las-travestis-Rada.pdf

Reisner, S. L., Conron, K. J., Tardiff, L. A., Jarvi, S., Gordon, A. R. \& Austin, S. B. (2014). Monitoring the health of transgender and other gender minority populations: Validity of natal sex and gender identity survey items in a U.S. national cohort of young adults. BMC Public Health, 14(1), artículo 1224. https://doi.org/10.1186/1471-2458-14-1224

Reisner, S. L., Hughto, J. M. W., Dunham, E. E., Heflin, K. J., Begenyi, J. B. G., Coffey-Esquivel, J. \& Cahill, S. (2015). Legal protections in public accommodations settings: A critical public health issue for transgender and gender-nonconforming people. The Milbank Quarterly, 93, 484-515. https://doi.org/10.1111/1468-0009.12127

Reisner, S. L., Pardo, S. T., Gamarel, K. E., Hughto, J. M. W., Pardee, D. J. \& Keo-Meier, C. L. (2015). Substance use to cope with stigma in healthcare among U.S. female-to-male trans masculine adults. LGBT Health, 2, 324-332. https://doi.org/10.1089/lgbt.2015.0001

Reisner, S. L., Poteat, T., Keatley, J., Cabral, M., Mothopeng, T., Dunham, E. ... Baral, S. D. (2016). Global health burden and needs of transgender populations: A review. The Lancet, 388, 412-436. https://doi.org/10.1016/S0140-6736(16)00684-X

Rigueiral, G. J. \& Seidmann, S. (2016, Noviembre). Análisis crítico de los principales estudios sobre las personas trans. Ponencia presentada en el VIII Congreso Internacional de Investigación y Práctica Profesional en Psicología, XXIII Jornadas de Investigación y XII Encuentro de Investigadores en Psicología del MERCOSUR, Facultad de Psicología, Universidad de Buenos Aires, Argentina. Extraído de https://www.aacademica.org/000-044/575.pdf

Rodríguez, C. (2018, Agosto). La biografía como ética política y feminista. Conferencia dictada en el Coloquio Internacional "Diversidades Sexuales y de Género: Debates Actuales", Universidad Alberto Hurtado, Santiago, Chile.

Roselló-Peñaloza, M. (2018). NO BODY: Clinical constructions of gender and transsexuality - pathologisation, violence and deconstruction. Abingdon, Reino Unido: Routledge.

Silva-Santisteban, A., Raymond, H. F., Salazar, X., Villayzan, J., Leon, S., McFarland, W. \& Caceres, C. F. (2012). Understanding the HIV/AIDS epidemic in transgender women of Lima, Peru: Results from a sero-epidemiologic study using respondent driven sampling. AIDS and Behavior, 16, 872-881. https://doi.org/10.1007/s10461-011-0053-5

Staples, J. M., Bird, E. R., Masters, T. N. \& George, W. H. (2018). Considerations for culturally sensitive research with transgender adults: A qualitative analysis. The Journal of Sex Research, 55, 1065-1076. https://doi.org/10.1080/00224499.2017.1292419

Stoller, R. (1964). A contribution to the study of gender identity. The International Journal of Psychoanalysis, 45, 220-226. Extraído de https://es.scribd.com/document/256126411/1964-A-contribution-to-the-study-of-gender-identity-docx

Stryker, S. (2017). Historia de lo trans. Las raíces de la revolución de hoy. Madrid, España: Continta Me Tienes.

Su, D., Irwin, J. A., Fisher, C., Ramos, A., Kelley, M., Rogel Mendoza, D. A. \& Coleman, J. D. (2016). Mental health disparities within the LGBT population: A comparison between transgender and nontransgender individuals. Transgender Health, 1, 12-20. https://doi.org/10.1089/trgh.2015.0001

Valdés, C. \& Riquelme, M. (2016). Personas LGBTI en Chile privadas de libertad, con libertades restringidas y otras situaciones de derechos humanos relacionadas con tortura, tratos o penas crueles, inhumanos o degradantes. Santiago, Chile: Frente de la Diversidad Sexual/Asociación Internacional de Lesbianas, Gays, Bisexuales, Trans e Intersex. Extraído de https://ilga.org/wpcontent/uploads/2016/02/SPT-Country-Visit-Chile-LGBT_ES.pdf

Valentine, D. (2007). Imagining transgender: An ethnography of a category. Durham, NC: Duke University Press.

World Health Organization (1992). International classification of diseases and related health problems. Genève, Suiza: Autor.

Fecha de recepción: Agosto de 2018.

Fecha de aceptación: Marzo de 2019. 